\title{
Effects of ibuprofen on exercise-induced muscle soreness and indices of muscle damage
}

\author{
A. E. Donnelly*1, PhD, R.J. Maughan ${ }^{1}$, PhD and P.H. Whiting ${ }^{2}$, PhD \\ ${ }^{1}$ Department of Environmental and Occupational Medicine and ${ }^{2}$ Department of Clinical Biochemistry, \\ Aberdeen University Medical School, Aberdeen
}

\begin{abstract}
Thirty-two volunteers participated in a two-period crossover study in which ibuprofen was tested against an identical placebo for its effectiveness in reducing muscle soreness and damage after two bouts of downhill running. Subjective soreness, quadriceps isometric strength and isometric endurance time at 50 percent of maximum strength, serum activities of creatine kinase, lactate dehydrogenase and aspartate transaminase and serum levels of creatinine and urea were recorded at intervals up to 72 hours after exercise. Each downhill run produced muscle soreness, and a decline in muscle strength and $\mathbf{5 0}$ percent endurance time, although these parameters were unaffected by ibuprofen treatment. All serum parameters measured increased after both runs, but for the three enzymes this increase was smaller after the second run. Serum creatine kinase and urea levels were higher in the ibuprofen group after both runs. These results indicate that ibuprofen is not an appropriate treatment for delayed onset muscle soreness and damage.
\end{abstract}

Keywords: Ibuprofen, delayed-onset muscle soreness, creatine kinase, urea

Unaccustomed exercise and exercise involving a large eccentric component can result in delayed onset muscle soreness (DOMS). This type of muscle soreness is generally not felt until eight or more hours following exercise, but may persist for three days ${ }^{1}$. Accompanying the muscle soreness is muscle weakness ${ }^{1,2}$ which persists long after the muscle soreness has receded. Damage to muscle fibres has also been observed following marathon running and eccentric exercise ${ }^{3,4}$ and after muscle lengthening in rats 5 .

Although DOMS is not a serious condition and can be prevented by prior training ${ }^{6}$, it may discourage further participation in exercise, and it is possible that muscle injury may result if heavy exercise is performed during the period of muscle weakness. No simple effective treatment is as yet available for DOMS. There is some evidence that DOMS may result from an inflammatory process which occurs in the muscle after exercise $e^{4,5,7}$. If this is the case, then it is possible that anti-inflammatory drugs may be effective in reducing DOMS and muscle damage.

Address for correspondence: Dr. R.J. Maughan, Department of Environmental and Occupational Medicine, University Medical School, Aberdeen AB9 2ZD, UK

* Present address: School of Health Sciences, Wolverhampton Polytechnic, Wolverhampton, UK

(C) 1990 Butterworth-Heinemann Ltd 0306-4179/90/030191-05
Although the majority of studies have found antiinflammatory drugs to be ineffective in reducing DOMS $^{8-10}$, a reduction in muscle soreness has been observed following treatment with aspirin ${ }^{11}$.

Ibuprofen is an over-the-counter non-steroidal anti-inflammatory drug now commonly used in the treatment of soft-tissue injuries ${ }^{12,13}$. The aim of the study described below was to test the effects of ibuprofen on muscle soreness, muscle weakness and muscle damage inferred from serum enzyme activity changes.

\section{Methods}

Forty healthy untrained male volunteers in the age range 18 to 30 years entered the study, which was approved by the joint ethical committee of Aberdeen University and Grampian Health Board. Each volunteer was required to perform two downhill runs lasting 45 minutes on an inclined motor-driven treadmill, with a ten week gap between runs. Ibuprofen or placebo were administered to each subject in a two period crossover design. Identical ibuprofen and placebo tablets were prepared and randomised by The Boots Company plc, Nottingham, England. Volunteers took two $600 \mathrm{mg}$ ibuprofen or placebo tablets 30 minutes before each bout of downhill running, and took one $600 \mathrm{mg}$ tablet every six hours up to 72 hours post-exercise. The total dose administered was therefore $8400 \mathrm{mg}$.

The running speed for each subject was assessed during an initial five minute run on the level. A three lead ECG was attached to each subject's chest prior to walking on the treadmill. The speed of the treadmill was gradually increased until a comfortable running speed for the subject was attained. Heart rate was monitored during this period, and, if necessary, the speed of the treadmill was adjusted so that a heart rate equivalent to 70 percent of the subject's estimated maximum (220 - subject's age) was attained. This was the speed at which both downhill runs were completed. A second five minute run on the level at the same speed as the first was performed prior to the second downhill run. Expired gas was collected during the last two minutes of each run on the level, and during two periods of two minutes in the downhill runs: from 19 to 21 minutes and from 39 to 41 minutes. Oxygen consumption for these periods was measured by a Gould $9000-\mathrm{IV}$ gas analysis system. 
Muscle soreness was assessed by questionnaire before and after each downhill run, and at $6,24,48$ and 72 hours thereafter. Soreness was rated on a scale of one (normal) to ten (very, very sore) for the following regions: front lower leg, back lower leg, front thigh, back thigh and buttocks. In addition, a fixed pressure of $6.2 \times 10^{4} \mathrm{Nm}^{-2}$ was applied at three sites in each region using a calibrated spring-loaded probe, and the soreness elicited by this pressure was recorded using the same rating scale. Hence a total of 20 soreness ratings were recorded on each soreness questionnaire.

Isometric muscle maximum voluntary contraction (MVC) strength was measured for the knee extensor muscles of each leg pre-exercise, and at 6, 24, 48 and 72 hours post-exercise. This was done with subjects seated on a chair similar to that described by Tornvall ${ }^{14}$. Tension produced by exerting force with the knee extensor muscles was recorded on a chart recorder placed in front of the subject. Isometric endurance time at 50 percent of MVC for the stronger leg was recorded at the same time intervals.

Blood samples were withdrawn from an anticubital vein pre- and post-exercise, and at $6,24,48$ and 72 hours after exercise. Serum was separated and frozen at $-20^{\circ} \mathrm{C}$ prior to analysis. Serum activity of the enzyme creatine kinase (CK) was measured on a Technicon RA 1000 (Technicon Ltd, Basingstoke, UK) random access analyser, using a Technicon test kit. Activities of the enzymes lactate dehydrogenase (LD) and aspartate transaminase (AST) were measured on a SMAC autoanalyser (Technicon Ltd, Basingstoke, UK) together with other serum biochemical parameters including creatinine and urea concentration.

All results are expressed as mean \pm SEM. Data in this study were analysed using repeated measures analysis of variance (ANOVA).

\section{Results}

\section{Demographic data}

Of the 40 volunteers, six were lost to the study in the ten week gap between the two downhill runs, one because of an unrelated sports injury, and five for personal reasons. A further two subjects were also eliminated from the study since one participated in strenuous exercise prior to the second downhill run, and the other missed the six hour sample point at the first period. Hence the statistical analysis was performed on two groups of 16 subjects. Group 1 received ibuprofen at the first period and placebo at the second period. Group 2 received placebo at the first period and ibuprofen at the second period. There was no difference between the groups for subjects' age (21 $\pm 1 \mathrm{yr}$, in both groups), or for weight, which at the start of the first period was $68.7 \pm 1.9 \mathrm{Kg}$ in group 1 , and $69.2 \pm 2.2 \mathrm{Kg}$ in group 2 .

\section{Oxygen consumption and running speed}

Running speed was $9.0 \pm 0.4 \mathrm{~km} \cdot \mathrm{h}^{-1}$ in the ibuprofen-placebo group, and $9.2 \pm 0.4 \mathrm{~km} . \mathrm{h}^{-1}$ in the placebo-ibuprofen group. As expected, oxygen consumption during downhill running was significantly less than that during runs on the level. There were no significant differences between ibuprofen and placebo treatments for oxygen consumption.

\section{Muscle soreness}

Total muscle soreness (the sum of all 20 soreness ratings on each soreness questionnaire) increased significantly after each exercise bout (Table 1), but there was no difference in soreness response between the two treatments, nor was there any difference in soreness response between the first and second downhill runs. When the individual muscle soreness measurements were examined separately, it was found that one measurement of the 20 (soreness induced by probing in the middle of the back thigh) demonstrated a treatment effect, with a greater increase in soreness in the ibuprofen group after both downhill runs $(p<0.05)$.

\section{Isometric strength and 50 percent endurance time}

Maximum voluntary contraction force for isometric contractions of the knee extensor muscles of the right and left legs fell after each exercise bout (Table 2). This decline in strength was never equivalent to more than 10 percent of initial leg strength, and was maximal 24 hours after exercise, with strength returning to normal levels by 72 hours post-exercise. Statistical analysis of the data revealed that for left leg MVC force, the group receiving ibuprofen at the first period and placebo at the second period demonstrated the greatest decline in strength irrespective of treatment.

Endurance time at 50 percent of MVC on the stronger leg also declined after exercise, reaching a minimum at six hours after exercise, but returning to normal by 72 hours after exercise (Table 3 ). Of the 32 volunteers whose MVC data were analysed, 30 used their right leg for measurement of endurance time, and only two used their left leg. The effect of excluding left leg data from the analysis was negligible. The group which received placebo at the first period and ibuprofen at the second period group demonstrated the greatest decline in strength, irrespective of treatment.

Table 1. Total soreness at the first and second periods of the study. The soreness values listed are the sum of 20 soreness ratings, and are on a scale of 20 (no soreness) to 200 (very, very sore at all points). Muscle soreness increased significantly $(p<0.01)$ after both downhill runs. Results are expressed as mean \pm SEM

\begin{tabular}{lccc}
\hline & & Ibuprofen & Placebo \\
First period & Pre & $23 \pm 1$ & $22 \pm 1$ \\
& $6 h$ & $31 \pm 3$ & $29 \pm 3$ \\
$24 h$ & $50 \pm 6$ & $29 \pm 5$ \\
$48 h$ & $42 \pm 4$ & $35 \pm 4$ \\
& $72 h$ & $30 \pm 2$ & $26 \pm 2$ \\
Second period & & Placebo & Ibuprofen \\
& Pre & $23 \pm 1$ & $22 \pm 1$ \\
& $6 h$ & $31 \pm 2$ & $29 \pm 2$ \\
& $24 h$ & $41 \pm 5$ & $38 \pm 5$ \\
& $48 h$ & $36 \pm 3$ & $36 \pm 5$ \\
& $72 h$ & $28 \pm 2$ & $29 \pm 3$ \\
\hline
\end{tabular}


Table 2. Maximum voluntary contraction force produced during isometric contraction of the left and right leg knee extensor muscles. Values are in Newtons, expressed as the mean $\pm \mathrm{SEM}$

\begin{tabular}{|c|c|c|c|c|c|}
\hline & \multicolumn{3}{|c|}{ Left leg } & \multicolumn{2}{|c|}{ Right leg } \\
\hline First period & $\begin{array}{l}\text { Pre } \\
6 h \\
24 h \\
48 h \\
72 h\end{array}$ & $\begin{array}{c}\text { Ibuprofen } \\
535 \pm 30 \\
530 \pm 24 \\
527 \pm 26 \\
546 \pm 29 \\
551 \pm 27\end{array}$ & $\begin{array}{c}\text { Placebo } \\
536 \pm 25 \\
505 \pm 21 \\
505 \pm 22 \\
513 \pm 21 \\
540 \pm 20\end{array}$ & $\begin{array}{c}\text { Ibuprofen } \\
584 \pm 27 \\
562 \pm 27 \\
553 \pm 30 \\
568 \pm 22 \\
583 \pm 24\end{array}$ & $\begin{array}{c}\text { Placebo } \\
567 \pm 24 \\
543 \pm 19 \\
539 \pm 20 \\
544 \pm 22 \\
566 \pm 25\end{array}$ \\
\hline Second period & $\begin{array}{r}\text { Pre } \\
6 h \\
24 h \\
48 h \\
72 h\end{array}$ & $\begin{array}{c}\text { Placebo } \\
551 \pm 21 \\
553 \pm 21 \\
550 \pm 22 \\
563 \pm 20 \\
563 \pm 24\end{array}$ & $\begin{array}{c}\text { Ibuprofen } \\
553 \pm 24 \\
540 \pm 25 \\
515 \pm 21 \\
525 \pm 29 \\
541 \pm 26\end{array}$ & $\begin{array}{c}\text { Placebo } \\
582 \pm 24 \\
540 \pm 25 \\
579 \pm 21 \\
597 \pm 22 \\
594 \pm 21\end{array}$ & $\begin{array}{c}\text { Ibuprofen } \\
594 \pm 23 \\
564 \pm 22 \\
555 \pm 24 \\
566 \pm 24 \\
581 \pm 28\end{array}$ \\
\hline
\end{tabular}

Isometric strength in both legs declined significantly after each downhill run $(p<0.01)$. Ibuprofen treatment did not significantly affect this strength loss. For the left leg, the decline in force was significantly smaller in the group receiving ibuprofen at the first period and placebo at the second period, irrespective of treatment $(p<0.01)$.

\section{Serum biochemistry}

Serum activities of CK, LD and AST increased after the downhill runs. Maximal activity of CK and AST was recorded 24 hours after exercise, while maximal LD activity was recorded six hours after each running bout. Serum levels of creatinine and urea also increased to a maximum at six hours after exercise. Increases in the activities of the three enzymes measured in the serum, CK, LD and AST were significantly lower at the second period than at the first $(\mathrm{p}<0.05)$.

Two of the serum parameters measured were affected by ibuprofen treatment. These were serum CK activity and serum urea level. These two parameters were significantly higher in the ibuprofen group than in the placebo group after both downhill runs (Figures 1 and 2).

Table 3. Endurance time at 50 percent of isometric maximal voluntary contraction force. This measurement was made on the stronger leg. Figures are in seconds, and the results are expressed as the mean \pm SEM

\begin{tabular}{lccc}
\hline & & Ibuprofen & Placebo \\
First period & Pre & $59 \pm 3$ & $62 \pm 3$ \\
& $6 h$ & $56 \pm 3$ & $55 \pm 3$ \\
$24 h$ & $58 \pm 2$ & $55 \pm 3$ \\
& $48 h$ & $55 \pm 2$ & $55 \pm 3$ \\
& $72 h$ & $59 \pm 2$ & $58 \pm 3$ \\
Second period & & Placebo & Ibuprofen \\
& Pre & $59 \pm 3$ & $59 \pm 3$ \\
& $6 h$ & $52 \pm 2$ & $52 \pm 2$ \\
& $24 h$ & $56 \pm 3$ & $54 \pm 4$ \\
& $48 h$ & $58 \pm 4$ & $57 \pm 3$ \\
& $72 h$ & $61 \pm 4$ & $61 \pm 4$ \\
\hline
\end{tabular}

Endurance time declined significantly after both downhill runs $(p<0.01)$. Ibuprofen treatment did not significantly affect the decline in endurance time. However, the decline in endurance time was smaller in the group receiving ibuprofen at the first period and placebo at the second period, irrespective of treatment $(\mathrm{p}<0.05)$.

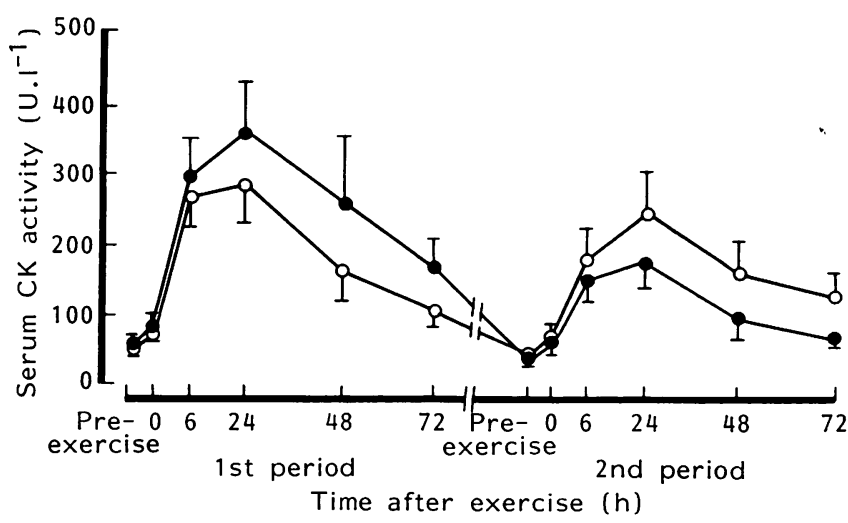

Figure 1. Serum creatine kinase activity for each group at both periods of the study. All points represent mean values \pm SEM. $=$ Ibuprofen - placebo group $(n=16)$; $\mathrm{O}=$ Placebo - ibuprofen group $(n=16)$. The serum CK activity increased significantly after each downhill run, although the increase at the second period was less than that at the first $(p<0.01)$. At both periods of the study this increase was significantly greater in the group receiving ibuprofen $(p<0.01)$

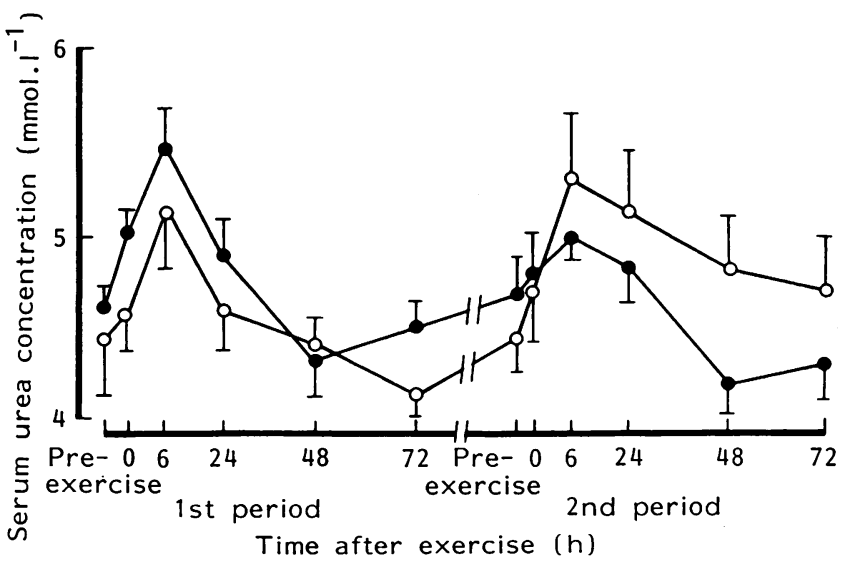

Figure 2. Serum urea concentration at the first and second periods of the study. All points represent mean values \pm SEM = Ibuprofen - placebo group $(n=16) ; 0=$ Placebo - ibuprofen group $(n=16)$. Serum urea concentration after each downhill run was significantly greater in the group receiving ibuprofen $(p<0.01)$ 


\section{Discussion}

It is evident from the results of this study that ibuprofen at this dosage is not effective in reducing DOMS after downhill running. This result concurs with previous studies ${ }^{8,10}$ in which similar nonsteroidal anti-inflammatory drugs were tested. These results suggest that DOMS may not be the result of inflammation.

Results from the MVC measurements indicate that the decline in force production after exercise was small, never exceeding ten percent of initial leg strength. This contrasts with strength losses of 50 percent or greater reported after eccentric arm exercise $^{15}$. If strength loss after eccentric exercise is related to damage to muscle fibres as has been observed in mice ${ }^{16}$, then the damage to muscle fibres after downhill running was not severe. However, the muscles used in the MVC measurement were not electrically stimulated, so it is not possible to assess the contribution of central and peripheral fatigue to the loss of force. Similarly, the decline in $\mathbf{5 0}$ percent endurance time was small but significant, indicating that the downhill run had affected the subjects' ability to sustain a muscle contraction.

The decline in muscle strength and in 50 percent endurance time did not differ between the first and second periods of the study, indicating that these indices of muscle function do not demonstrate the long repeated bout effect observed for muscle enzyme release. It is thus possible that muscle strength loss following this type of exercise is not directly related to the release of enzymes from damaged muscle fibres. Muscle soreness also reached the same level after both exercise bouts, again indicating that muscle enzyme release and muscle soreness may be unrelated.

The results indicate that the response to ibuprofen treatment observed for both left leg strength and 50 percent endurance time on the stronger leg differed between the two groups. However, this result may be the product of an accident of selection, whereby subjects in the ibuprofen-placebo group were less susceptible to long term muscle fatigue than subjects in the placebo-ibuprofen group.

Perhaps the most surprising result of the whole study was the finding that after downhill running, the serum activity of CK and serum urea level were greater in the subjects receiving ibuprofen than in subjects receiving placebo. It is possible that the observed results reflected increased levels of muscle damage in the ibuprofen treated subjects, since serum CK activity increases can be related to skeletal muscle damage. However, no significant differences were detected between ibuprofen and placebo groups for the enzymes LD and AST, which also increase following muscle damage, although this could be because these enzymes are less sensitive indicators of muscle damage than CK. Similarly, the observed difference in serum urea concentration between groups was not accompanied by differences in serum creatinine levels.

The apparent increase in serum $\mathrm{CK}$ activity and urea concentration could perhaps result from action of ibuprofen on plasma volume and plasma protein content, since it has been observed that ibuprofe. treatment decreased plasma protein content immediately after exercise ${ }^{17}$. It may be that prolonged ibuprofen treatment after exercise produces further changes in plasma protein content. Again, this would not explain why the other serum proteins measured were not affected by ibuprofen treatment.

Clearly, the treatment of DOMS with ibuprofen at this dosage is not beneficial, since it reduces neither muscle soreness nor muscle strength loss. The effect of ibuprofen on the serum parameters measured suggests that ibuprofen treatment further increases circulating levels of $\mathrm{CK}$ and urea. The mechanism of this increase cannot be ascertained without further studies.

\section{Acknowledgements}

This study was supported by the Boots Company, plc. A.E Donnelly was supported by a University of Aberdeen Medical Endowments Research Sudentship, and R.J. Maughan is supported by a grant from Shell Expro (UK) Ltd. The authors wish to thank the operators of the SMAC system in the Department of Clinical Biochemistry.

\section{References}

1 Newham, D.J., Jones, D.A. and Edwards, R.H.T. Large delayed plasma creatine kinase changes after stepping exercise Muscle Nerve 1983, 6, 380-385

2 Newham, D.J., Jones, D.A. and Clarkson, P.M. Repeated high-force eccentric exercise: effects on muscle pain and damage J Appl Physiol 1987, 63, 1381-1386

3 Friden, J., Sjostrom, M. and Ekblom, B. Myofibrillar damage following intense eccentric exercise in man Int J Sports Med 1983, 4, 1701-6

4 Hikida, R.S., Staron, R.S., Hagerman, F.C., Sherman, W.M. and Costill, D.L. Muscle fibre necrosis associated with human marathon runners J Neurol Sci 1983, 59, 185-203

5 Stauber, W.T., Fritz, V.K., Vogelbach, D.W. and Dahlmann, B. Characterization of muscles injured by forced lengthening - Cellular infiltrates Med Sci Sports Exerc 1988, 20, 345-353

6 Byrnes, W.C., Clarkson, P.M., Hsieh, S.S., Frykman, P.N. and Maughan, R.J. Delayed onset muuscle soreness following repeated bouts of downhill running J Appl Physiol 1985, 59, 710-715

7 Smith, L.L., McCammon, M., Smith, S., Chamness, M., Israel, R.G. and O'Brien, K.F. Leukocytosis: response to metabolic demands or a sign of acute inflammation related to delayed muscle soreness (DMS) Med Sci Sports Exerc 1988, 20(2), S74

8 Donnelly, A.E., McCormick, K., Maughan, R.J., Whiting, P.H. and Clarkson, P.M. Effects of a non-steroidal anti-inflammatory drug on delayedonset muscle soreness and indices of damage Brit $J$ Sports Med 1988, 22, 35-38

9 Headley, S.A.E., Newham, D.J. and Jones, D.A. The effect of prednisilone on exercise induced muscle soreness and damage Clin Sci 1985, 10(13), 85

10 Kuipers, H., Keizer, H.A., Verstappen, F.T.J. and Costill, D.L. Influence of a prostaglandin-inhibiting drug on muscle soreness after eccentric work Int J Sports Med 1985, 6, 336-339 
11 Bansil, C.K., Wilson, G.D. and Stone, N.H. Role of prostaglandins $E$ and $F_{2}$ alpha in exercise induced delayed muscle soreness Med Sci Sports Exerc 1985, 17, 276

12 McLatchie, G.R., Allister, C., MacEwen, C., Hamilton, G., McGregor, H., Colqihon, I. and Pickvance, N.J. Variable schedules of ibuprofen for ankle sprains Brit J Sports Med 1985, 19, 203-206

13 Muckle, D.S. Comparative study of ibuprofen and aspirin in soft tissue injuries Rheum Rehabil 1984, 13, 141-147

14 Tornvall, G. Assessment of physical capacities with special reference to the evaluation of maximal voluntary isometric muscle strength and maximal working capacity Acta Physiol Scand 1963, 50, (Suppl.) 201

15 Clarkson, P.M. and Tremblay, I. Exercise-induced muscle damage, repair and adaptation in humans $J$ Appl Physiol 1988, 65, 1-6

16 McCulley, K.K. and Faulkner, J.A. Injury to skeletal muscle fibres of mice following lengthening contractions J Apple Physiol 1985, 59, 119-126

17 Pivarnik, J., Kayrouz, T., Senay, L.C. Plasma volume and protein content in progressive exercise: influence of cyclooxygenase inhibitors Med Sci Sports Exerc 1985, 17, 1531-6 